\title{
Inversion de l'asymétrie droite-gauche chez des souris transgéniques
}

Alors que la mise en place d'axes dans l'embryon est essenticlle pour le développement, les factcurs moléculaires de la polaritć restent inconnus, et plus cncore ceux qui sont responsables de l'asymétric droite-gauche. Celle-ci se reflète dans la position des viscères estomac, foic, ratc, cour. On a décrit des mutations qui provoquent unc inversion des organes chez l'homme et la souris ; ces mutants entraînent une inversion de la polarité environ une fois sur deux, et on $\mathrm{cn}$ a déduit qu'il n'existc pas dc "commande centralc" rigourcuse pour la polarité droitegauche. Cette conclusion est aujourd'hui bousculéc par unc découverte fortuitc faite par unc équipc américaine dirigéc par P. A. Overbeck (Houston TX, USA) [1]. Cette ćquipe s'ćtait lixé comme objectif d'ćtudier les effets du transfert par transgenc̀se d'un gène de tyrosinase (la premic̀rc enzyme de la voie des mélanines) dans unc souchc de souris albinos, FVB/N [2]* Plusicurs "familles" de souris transgéniques furent ainsi obtenucs en injectant un minigène, dit Ty811C, dans des embryons au stade unicellulaire. A l'état hémizygote (une dose de transgène), ces souris se reconnaisscnt, à la naissance, par unc pigmentation oculaire, ct, à l'âge adulte, par un pclage brun clair. On chercha ensuite à produire des homozygotes par union de ces hémizygotes. Or, dans une "famille ", OVE210, il fut impossible d'obtenir des adultes. Ces animaux meurent dans les sept jours après la naissance. En disséquant ces souris mutécs, on eut la surprise de trouver l'estomac ct la rate à droite de l'abdomen ; l'orientation du cœur, des poumons et du foic était ćgalement inversée. En examinant 74 foctus avant la naissance, on trouva $22 \%$ d'albinos, $55 \%$ de pigmentés à polarité normale,

* Le caractère albinos résulte d'une mutation dan. l'enzyme tyrosinase, le remplacement d'une glycine par une sćrine en position 103 /2].
$23 \%$ de pigmentés avec situs inversus, pourcentages typiques d'une mutation récessive.

A partir d'une banque génomique d'ADN provenant des souris OVE210, on obtint des clones contenant le site d'intégration du transgène. On put ainsi distinguer les souris mutantes et normales, et confirmer que les animaux à situs inversus étaient homozygotes pour le transgène.

Il est à noter que dix autres familles ont été obtenues à l'aide du même minitransgène Ty811C : aucune ne présente de situs inversus; le facteur en cause est donc bien le licu d'insertion, c'est-à-dire un phénomènc de mutagenc̀se inscrtionnelle. L'observation faite par Yokoyama et al. [1] n'est pas sans précédent : comme nous l'avons mentionné plus haut, on connaissait depuis trente ans une mutation iv (inversus viscerum), et il était nécessaire de vérificr si cette dernière et la mutation insertionnelle inv n'étaient pas alléliques. Cette possibilité fut exclue par des croisements entre des souris homozygotes $i v$ et des hémizygotes inv ; tous les descendants avaient polarité et viabilité normales. Cette absence d'allélisme est corroborée par les localisations chromosomiques: le locus iv est porté par le chromosome 12 [3] ; le locus inv a pu ĉtre localisé à la partie proximale du chromosome 4, à proximité de l'oncogène $v$-mos, des gènes de la sousunité $\alpha$ de l'hormone thyréotrope (Tsha) et de l'hexabrachion ou ténascine $(H x b)$ [1].

Ces résultats conduisent à de nouvelles questions [4]. Tout d'abord, la raison pour laquelle ces animaux ne peuvent dépasser une semaine n'est pas évidente. Bien que porteurs d'inversion viscérale, ils ne semblent pas mourir de troubles cardiaques ou digestifs, mais de défaillance des reins, organes symétriques. Le point le plus intéressant de la discussion reste le mécanisme de production du situs inversus. La mutation est récessive, et les affec- tions récessives résultent en règle d'une perte de fonction. On est donc conduit à attribuer au gène inv un rôle actif dans la polarité normale ; en l'absence de directives de sa part, une voie défective instaurerait une polarité inverse. On connaît certaines des étapes de la rotation qui conduit les embryons de la position lordotique à la latéralité, vers le $9^{e}$ jour embryonnaire. Les auteurs ont pu vérifier que chez les animaux homozygotes pour le transgène, la rotation s'effectuait en sens inverse, d'après la position des vaisseaux vitellins que l'on retrouvait du côté droit de leur corps au lieu du côté gauche comme il est habituel.

Est-il possible enfin de tirer de cette découverte des enseignements pour la pathologie humaine ? En 1935 a été décrit (in [1]) un syndrome dit de Kartagener, récessif autosomique avec situs inversus, accompagné de troubles respiratoires et de stérilité masculine. Sa localisation chromosomique n'est pas connue. Des gènes proches du locus inv de la souris, nous avons noté que chez l'homme, le proto-oncogène MOS (homologue du v-mos murin) est situé en 8q11, et Hxb est en 9q32-34, alors que TSHA n'est pas encore localisé à notre connaissance.

J.-C. D.

1. Yokoyama T, Copeland NG, Jenkins NA Montgomery CA, Elder FFB, Overbeek PA Reversal of left-right asymmetry : a situs inversus mutation. Science $1993 ; 260: 679-82$.

2. Yokoyama $T$, Silversides DW, Waguiere $K G$ Kwon BS, Takeuchi T, Overbeek PA. Conserved cysteine to serine mutation in tyrosinase is responsible for the classical albino mutation in laboratory mice. Nucleic Acids Res $1990 ; 18$ 7293-8.

3. Brueckner M, D'Eustachio P, Horwich AL Linkage mapping of a mouse gene, $i v$, that controls left-right asymmetry of the heart and viscera. Proc Natl Acad Sci USA 1989 ; 86 : 5035-8. 4. Ewing T. Genetic " master switch " for leftright symmetry found. Scince $1993 ; 260: 624-5$. 